\title{
Variabilidade Espaço-Temporal do Monóxido de Carbono Sobre a América do Sul a Partir de Dados de Satélite de 2003 A 2012
}

\author{
Yara Luiza Farias dos Santos ${ }^{1}$, Rodrigo Augusto Ferreira de Souza ${ }^{2}$, \\ Jaidete Monteiro de Souza ${ }^{2}$, Rita Valéria Andreoli ${ }^{2}$, Mary Toshie Kayano ${ }^{3}$, \\ Igor Oliveira Ribeiro ${ }^{1}$, Patrícia Costa Guimarães ${ }^{1}$ \\ ${ }^{1}$ Programa de Pós-Graduação em Clima e Ambiente, Instituto Nacional de Pesquisas da \\ Amazônia e Universidade do Estado do Amazonas, Manaus, AM, Brasil. \\ ${ }^{2}$ Escola Superior de Tecnologia, Universidade do Estado da Amazonas, Manaus, AM, Brasil. \\ ${ }^{3}$ Centro de Previsão de Tempo e Estudos Climáticos, Instituto Nacional de Pesquisas Espaciais, \\ São José do Campos, SP, Brasil.
}

Recebido: 16 de Dezembro de 2015 - Aceito: 17 de Maio de 2016

\begin{abstract}
Resumo
O presente trabalho investiga o comportamento do monóxido de carbono (CO) troposférico sobre a região central da América do Sul e sua variabilidade espaço-temporal usando informações do sistema de observações da terra do satélite AQUA (EOS/AQUA, em inglês) no período de 2003 a 2012. Os resultados mostram um comportamento sazonal bem definido da concentração de $\mathrm{CO}$, com redução na estação chuvosa e aumento na estação seca, devido ao aumento da queima de biomassa nesse período. Como a queima de biomassa está diretamente associada à variabilidade climática, ou seja, à diminuição/aumento de chuvas na parte central e leste da América do Sul, o CO possui uma maior variabilidade sobre o Brasil Central, região esta que apresenta o maior número de focos de queima. Os resultados mostram também que a variabilidade de CO na escala interanual está relacionada ao fenômeno El Niño/Oscilação Sul (ENOS), de modo que a diminuição (aumento) de chuvas na parte central e leste da América do Sul durante a fase inicial do evento de La Niña (El Niño) contribui para aumentar (diminuir) os focos de queimada e consequentemente, as emissões de CO nesta região. Por outro lado, durante a fase madura do ENOS, as maiores variabilidades de precipitação e concentração de CO acontecem nas regiões norte e nordeste da América do Sul. Os resultados apresentados neste trabalho podem ser úteis para atividades de monitoramento.
\end{abstract}

Palavras chave: monóxido de carbono, queima de biomassa, variabilidade climática, El Niño-Oscilação sul.

\section{Spatio-Temporal Variability of Carbon Monoxide Over South America Using Satellite-Sensed Data from 2003 to 2012}

\begin{abstract}
Using the information of the Earth Observation System/AQUA (EOS/AQUA) satellite during the 2003-2012 period, the present work investigates the tropospheric carbon monoxide (CO) behavior over the central South American region, and its spatiotemporal variability. The results show a well-defined seasonal behavior of the CO concentration, with decrease during the wet season and increase during dry season, due to the biomass burning increase during this period. Since the biomass burning is directly associated with the climate variability, through the rainfall decrease/increase in the central and eastern South American sector, the CO possesses high variability in central Brazil, the region with the largest number of burning focus. The results also show that the $\mathrm{CO}$ variability in the interanual time scale is related to the $\mathrm{El}$ Niño/Southern Oscillation (ENSO) phenomenon, such that rainfall reduction (increase) in the central and eastern part of South America during the La Niña (El Niño) onset phase contributes to increase (reduce) the biomass burning and in consequence the $\mathrm{CO}$ emission in this region. On the other hand, during the ENSO mature stage, the great rainfall and CO concentration variabilities occur in the northern and northeastern South American regions. The results presented in this paper might be useful for monitoring activities.
\end{abstract}

Keywords: carbon monoxide, biomass burning, climate variability, El Niño-Southern oscillation.

Autor de correspondência: Yara L. F. dos Santos, yarasantos88@gmail.com. 


\section{Introdução}

Monóxido de Carbono ( $\mathrm{CO}$ ) é um gás traço muito importante na química da atmosfera, pois atua como sumidouro do radical hidroxila $(\mathrm{OH})$, o que influencia diretamente as atividades oxidantes da atmosfera, e é o principal precursor do dióxido de carbono $\left(\mathrm{CO}_{2}\right)$. A redução das concentrações de $\mathrm{OH}$ na atmosfera, por sua vez, afeta o tempo de residência de outros gases, principalmente o metano $\left(\mathrm{CH}_{4}\right)$ e dióxido de enxofre $\left(\mathrm{SO}_{2}\right)$ (Liou, 2002). Além disso, o CO é o maior precursor do ozônio troposférico, poluente presente nas camadas mais baixas da atmosfera, e é usado como indicador de atividades humanas uma vez que sua origem é principalmente a queima incompleta de carbono, devido à mudança de uso da terra como a substituição de florestas e savanas por agricultura e pastagem (Wotawa et al., 2001; Langenfelds et al., 2002; Novelli et al., 2003; D'Amelio, 2006; Edwards et al., 2006a; Duncan e Logan, 2008; McMillan et al., 2008; Yurganov et al., 2008; Rajab et al., 2011; Liu et al., 2013). Por possuir um tempo de residência na atmosfera relativamente curto o $\mathrm{CO}$ é frequentemente usado para estudar processos de transporte de gases (Liou, 2002; Susskind et al., 2003; McMillan et al., 2005; Edwards et al., 2006a; Wallace e Hobbs, 2006; Yurganov et al., 2008; Rajab et al., 2011; Andreae et al., 2012; Thonat et al., 2012).

Importantes instrumentos que auxiliam na inferência da concentração de gases traços e gases de efeito estufa (GEE) presentes na atmosfera são os sondadores a bordo de satélites. A plataforma AQUA, lançada em 2002 pela NASA (do inglês, National Aeronautics and Space Administration), possui cobertura global diária e varredura horizontal de $1650 \mathrm{~km}$ de largura, e conta com sensores em microondas que operam em conjunto com o sensor AIRS (do inglês, Atmospheric Infrared Sounder), os quais auxiliam no processo de inferência de perfis atmosféricos mesmo em condições de nebulosidade parcial. Com isso, é possível criar um mosaico de $\mathrm{CO}$ em menos tempo do que àqueles criados a partir de outros sensores lançados anteriormente e ainda estudar fenômenos de escala diária. O sensor AIRS, utilizado neste trabalho foi o primeiro sondador avançado de radiação infravermelha (Parkinson, 2003), com seus 2378 canais espectrais cobrindo a banda espectral do infravermelho (IV), de 3,7 a $15,4 \mu \mathrm{m}$, e representou o avanço mais importante na tecnologia de sensoriamento remoto por satélites ambientais (Aumann e Pagano, 1994; Aumann e Miller, 1995; Morse et al., 1999; Fishbein et al., 2003). Os diversos canais espectrais fornecem dados de vários parâmetros atmosféricos, bem como perfis de vapor d'água, nuvens, poeira e gases-traço como $\mathrm{CO}_{2}, \mathrm{CO}, \mathrm{SO}_{2}, \mathrm{O}_{3}$ e $\mathrm{CH}_{4}$, fornecendo observações globais (Aumann e Miller, 1995; Rodgers, 1998; Morse et al., 1999; Chahine e Fetzer, 2000; Fishbein et al., 2003; Susskind et al., 2003).

Em seu estudo, McMillan et al. (2005) mostraram a possibilidade de utilizar o sensor AIRS para estimar a concentração de CO na média troposfera e monitorar possíveis variabilidades diárias e transporte da concentração deste gás. Em seus resultados, comparações preliminares com perfis de aeronaves in situ indicaram que as estimativas de $\mathrm{CO}$ do AIRS estavam próximas da meta de precisão de $15 \%$ estabelecida por meio de simulações de pré-lançamento.

De fato, trabalhos posteriores (McMillan et al., 2007; Yurganov et al., 2008; Rajab et al., 2011; Liu et al., 2013) demonstram a utilidade das observações de CO por satélite na troposfera para estudos de qualidade do ar e uso da terra, uma vez que as principais fontes de CO para a atmosfera são a combustão incompleta do carbono pela queima de combustíveis fósseis e a substituição de florestas e savanas por agricultura e pastagem. Esta última é a principal causa das emissões brasileiras (Hooghiemstra et al., 2012). O transporte de $\mathrm{CO}$ e seu alto grau de dependência sazonal, muitas vezes associado a eventos de queima de biomassa, bem como a fenômenos que ocorrem na escala interanual ilustram a necessidade de monitoramento contínuo, uma vez que, as observações mensais e sazonais climatológicas suavizam os casos de máxima ou mínima emissão em qualquer época do ano. Ainda, a correspondência da variabilidade interanual de CO com os eventos El Niño-Oscilação Sul (ENOS), como demonstrada em diversos estudos (Edwards et al., 2006b; McMillan et al., 2007; Hooghiemstra et al., 2012; Thonat et al., 2012), fornece um exemplo do impacto da variabilidade do clima nos níveis de poluição. Outros fatores como, por exemplo, a pressão de preços internacionais de commodities e o desenvolvimento de culturas mais produtivas, podem também influenciar o número de focos de incêndio e desempenhar alguma influência na variabilidade do CO. Relações entre os mercados agrícolas internacionais e o destino da floresta Amazônica estão documentadas em trabalhos anteriores (Fearnside, 2005; Malhi et al., 2008). No entanto, para análises de curtos períodos é difícil isolar o impacto direto da atividade artificial das alterações climáticas. Assim, essas análises estão fora do escopo desse trabalho.

Neste contexto, esse estudo busca as possíveis relações entre a concentração/variabilidade desse gás, alterações climáticas e focos de queimadas, considerando o período de 10 anos de dados remotos de CO sobre a América do Sul. Além do interesse científico, deve-se ressaltar que o melhor conhecimento das emissões de gases é fundamental para adoção de políticas públicas que reduzam queimadas e desmatamentos, sendo esta última a maior fonte de emissões brasileiras.

\section{Dados e Metodologia}

Foram utilizadas as estimativas das concentrações médias semanais do conteúdo de CO integrado verticalmente entre superfície e topo da atmosfera a partir do sistema de sondagem AQUA (versão 5). Os dados encontram-se no formato HDF (Hierarchical Data Format) com 
resolução espacial de $1^{\circ}$ em latitude e longitude. A área de estudo compreende uma ampla região da América do Sul limitada em $5^{\circ} \mathrm{N}, 35^{\circ} \mathrm{S}, 35^{\circ} \mathrm{W}$ e $75^{\circ} \mathrm{W}$. Os dados representam uma média a cada 8 dias das estimativas de $\mathrm{CO}$ e a série de dados compreendeu o período de 2003 a 2012. Estes dados foram obtidos no portal da NASA. Foi utilizado ainda, o número mensal de focos de queimadas detectados pelos satélites NOAA, TERRA/AQUA e GOES para a região da América do Sul, disponível no portal eletrônico de Monitoramento de Queimadas e Incêndios do Instituto Nacional de Pesquisas Espaciais (INPE). O período corresponde aos 10 anos estudados nessa pesquisa. Também foi usada a versão 6 da reanálise mensal de precipitação do GPCC (Global Precipitation Climatology Centre), com resolução espacial de $1^{\circ}$ em latitude e longitude, para o período entre 1901 e 2010 (Schneider et al., 2008). Os dados foram extraídos sobre a América do Sul, situada entre $10^{\circ} \mathrm{N}-45^{\circ} \mathrm{S}$ e $85^{\circ} \mathrm{W}-25^{\circ} \mathrm{W}$, para o período de 2003 a 2010.

Os comportamentos médios anual e sazonal do $\mathrm{CO}$ foram analisados considerando a distribuição latitudinal da concentração de $\mathrm{CO}$ sobre a área de estudo obtida pela média zonal deste parâmetro nesta área em função do tempo. Além disso, foram construídas séries de anomalia semanais de $\mathrm{CO}$, contendo as médias espaciais das anomalias da concentração de $\mathrm{CO}$ integrada verticalmente na faixa latitudinal sobre a América do Sul, entre $20^{\circ} \mathrm{S}$ a $5^{\circ} \mathrm{S}$ e entre as longitudes de $35^{\circ} \mathrm{W}$ a $75^{\circ} \mathrm{W}$, considerando as médias semanais do período de 2003-2012. Da mesma maneira, anomalias de $\mathrm{CO}$ semanais normalizadas pelo desvio padrão foram obtidas em cada ponto de grade. Para o cálculo de anomalias para o conjunto de dados de focos de queima e precipitação, o ciclo anual foi determinado pelo cálculo das médias mensais.

Para estudar a variabilidade espaço-temporal do $\mathrm{CO}$ sobre a América do Sul as séries da concentração de $\mathrm{CO}$ em cada ponto de grade foram filtradas para as escalas semianual ( 3 a 7 meses) e interanual ( 1 a 4 anos), utilizando-se a transformada de ondeleta como filtro passa-banda conforme formulação definida por Torrence e Compo (1998). A escolha destas escalas de variabilidade decorre do fato que as altas emissões de $\mathrm{CO}$ pelas queimadas apresentam um ciclo semianual bem característico. Por outro lado, a variabilidade climática dominante na região tropical ocorre em escalas de tempo interanual, associada ao ENOS.

Em seguida, foi empregada a técnica de análise das Funções Ortogonais Empíricas (EOF, do inglês Empirical Orthogonal Function) nas séries de anomalia de concentração de CO filtradas nas escalas semianual e interanual, com o propósito de encontrar os padrões dominantes em cada escala de variabilidade. Análises de EOF foram feitas separadamente para cada escala de variabilidade. Os cálculos de EOF foram baseados na matriz correlação. Os autovetores correspondentes às análises de EOF são apresentados como padrões de correlações. Para as discussões de EOF, assume-se que para amplitudes positivas dos mo- dos, o sinal das correlações corresponde ao sinal das anomalias. Detalhamentos da técnica de EOF podem ser encontrados em Kutzback (1976).

Para obter a relação desses padrões com a variabilidade de focos de queima e precipitação, o mesmo procedimento de filtragem de dados foi realizado para essas séries. Análises qualitativas foram realizadas das relações entre as variações de focos de queima, precipitação e de CO. Campos espaciais das anomalias de $\mathrm{CO}$ e precipitação para dois anos contrastantes, selecionados a partir das séries de componentes principais obtidas nas análises de EOF, também são apresentados.

\section{Resultados e Discussão}

\subsection{Variação sazonal}

A Fig. 1 mostra a variação temporal da concentração de $\mathrm{CO}$ integrado na atmosfera em função da latitude. Observa-se um ciclo sazonal bem definido, o que concorda com os resultados mostrados em trabalhos anteriores (Connors et al., 1999; Langenfelds et al., 2002; Edwards et al., 2006a e 2006b; Andreae et al., 2012; Hooghiemstra et al., 2012). Máximos valores de emissão de $\mathrm{CO}$ entre as latitudes de $5^{\circ} \mathrm{S}$ e $20^{\circ} \mathrm{S}$ refletem as altas emissões provenientes da queima nas florestas da Amazônia e do cerrado durante o período de setembro e outubro. Os picos menores, nas latitudes entre $5^{\circ} \mathrm{S}$ e $5^{\circ} \mathrm{N}$, a partir de janeiro a março de cada ano são resultados dos incêndios que ocorrem nas regiões da Venezuela e Colômbia e da queima de biomassa no oeste da África (Edwards et al., 2006a). Notase ainda, uma variação interanual dos valores de máxima concentração de $\mathrm{CO}$, de modo que os maiores valores são observados nos anos de 2004, 2005, 2007 e 2010, e os menores valores, nos anos de 2003, 2008, 2009 e 2011 ocorrem entre as latitudes de $5^{\circ} \mathrm{S}$ e $20^{\circ} \mathrm{S}$ (Fig. 1). Anomalias positivas (negativas) mais acentuadas nos anos de 2005, 2007 e 2010 (2009 e 2011) coincidem com os anos em que ocorreram máximos valores positivos (negativos) de anomalias de foco de queimadas como mostrado na Fig. 2. Nessa figura pode-se observar qualitativamente uma concordância entre as anomalias de $\mathrm{CO}$ e focos de queimada. Embora seja possível detectar focos de queimada através de observações de satélite, cautela deve ser tomada quando usamos o número de focos para inferir emissões. Em adição, os valores de CO sobre a América do Sul, podem representar uma combinação da emissão local e àquela transportada de outras regiões (Edwards et al., 2006a).

Por outro lado, estudos anteriores mostram que a variabilidade climática pode explicar variações interanuais na concentração de $\mathrm{CO}$ na atmosfera. A Fig. 3 mostra a relação entre a série de precipitação média sobre a região da América do Sul, entre as latitudes de $5^{\circ} \mathrm{S}$ a $20^{\circ} \mathrm{S}$ e a série de concentração de CO sobre essa região. Particularmente, sobre os períodos de máximos valores positivos (negativos) 


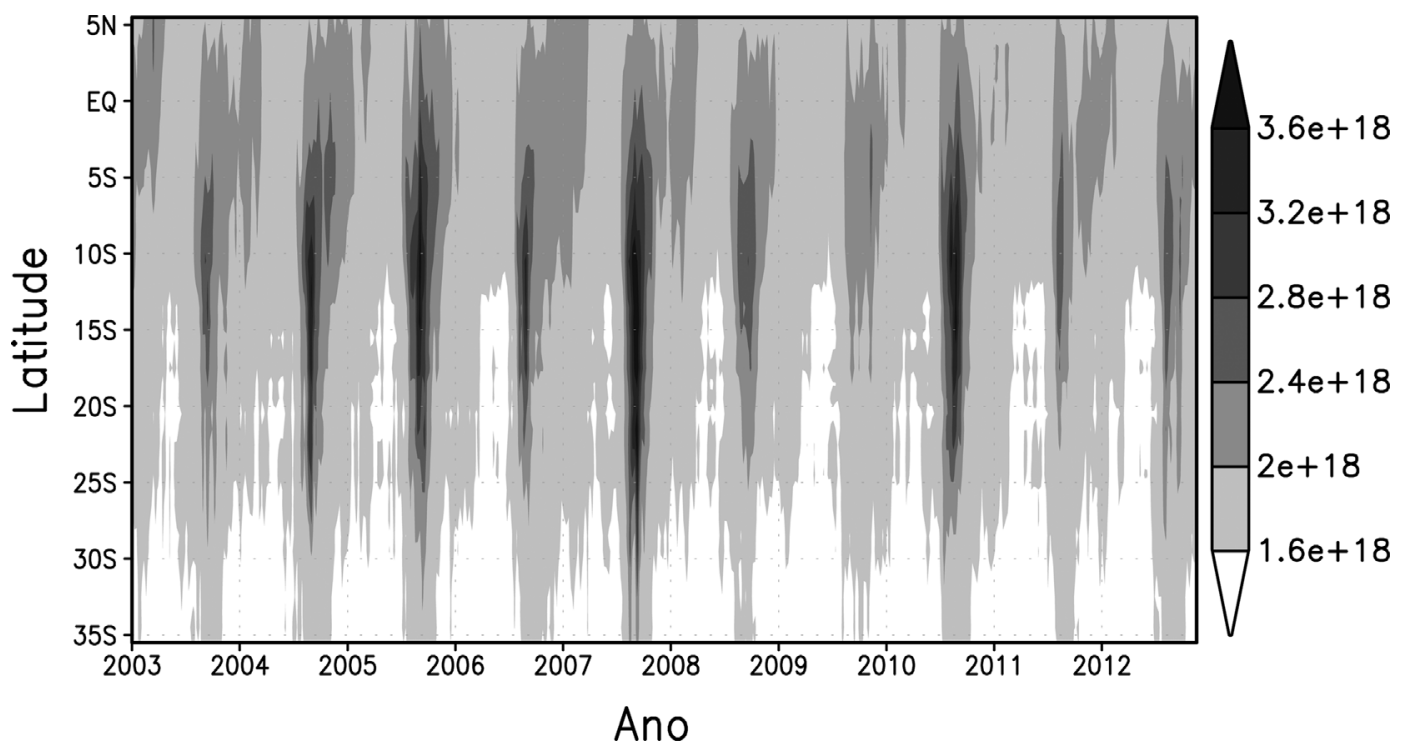

Figura 1 - Concentração média zonal de $\mathrm{CO}\left(\mathrm{molec} / \mathrm{cm}^{2}\right)$ sobre a América do Sul.

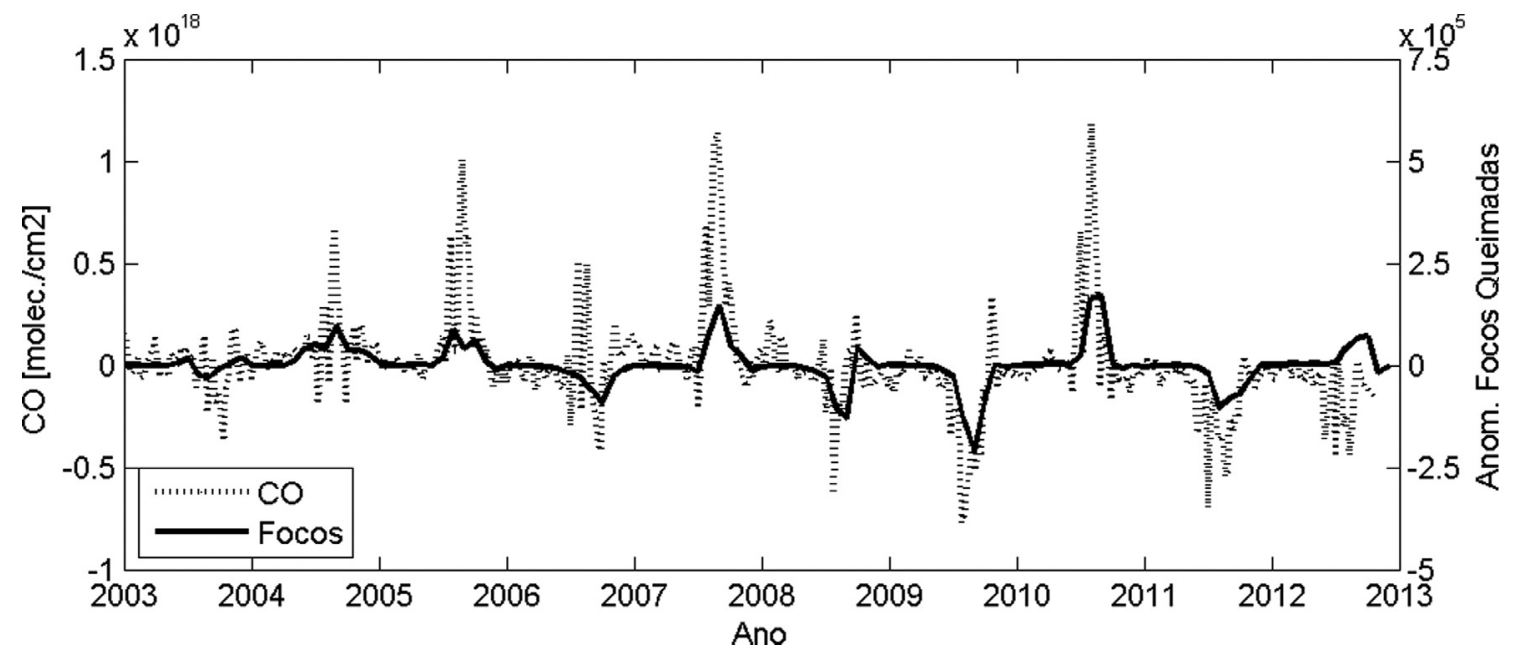

Figura 2 - Séries de anomalias de concentração de CO (linha pontilhada) e anomalias de focos de queimadas (linha contínua) sobre a América do Sul.

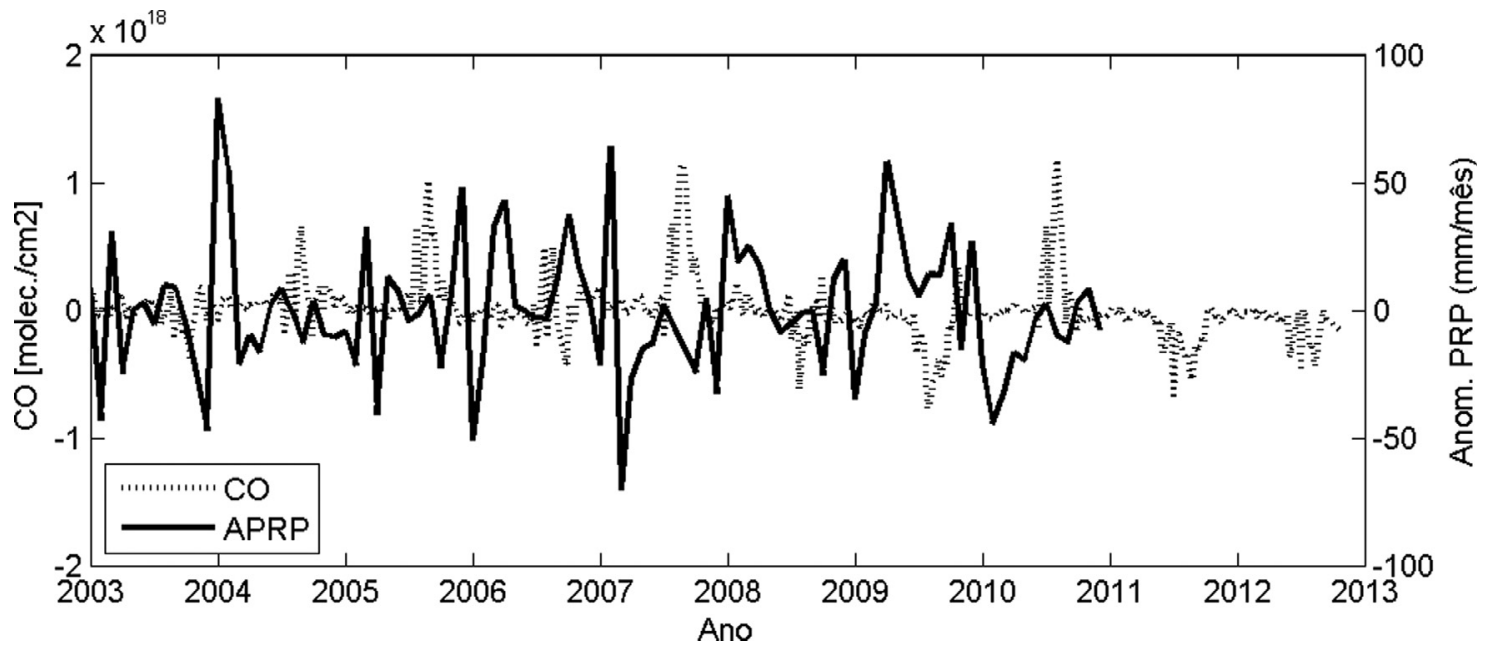

Figura 3 - Séries de anomalias de concentração de $\mathrm{CO}$ (linha pontilhada) e anomalias de precipitação (linha contínua) sobre a América do Sul, entre $5^{\circ} \mathrm{S}$ e $20^{\circ} \mathrm{S}$. 
de anomalias de CO, como por exemplo, os anos de 2005, 2007 e $2010(2008,2009)$ ocorrem máximos valores negativos (positivos) de precipitação. De acordo com Torres et al. (2010), o grande número (baixa incidência) de focos de queima em 2007 (2009) foi propiciado pelo déficit (excesso) de precipitação em torno de $30 \%$ (34\%), que afetou a América do Sul.

\subsection{Variabilidades semianual e interanual}

Os primeiros dois modos de variabilidade de $\mathrm{CO}$ na escala de tempo semianual, apresentados nas Figs.4a e 5a, explicam $49,9 \%$ e $11 \%$ da variabilidade total de CO contida nesta escala, respectivamente. O primeiro modo é dominado por variações que ocorrem sobre toda a área de estudo, com máximo na região central desta área com valor de correlação acima de 0,8 . Uma vez que o quadrado das correlações equivale à variância explicada localmente, este modo explica aproximadamente $64 \%$ da variância nessa região. A primeira componente principal (CP01, Fig. 4c) exibe uma oscilação semianual com valores positivos (negativos) ocorrendo nos meses de março a maio (setembro a outubro), com os máximos negativos nos anos de 2005, 2007 e 2010. Para o período de setembro a outubro (estação seca), o padrão de variabilidade mostra um aumento do total de concentração de CO. O segundo modo (Fig. 5a) é caracterizado por um padrão dipolar com máximas correlações positivas (negativas) na região norte da América do Sul, sobre as regiões das Guianas e Suriname (estados de Mato Grosso do Sul, São Paulo e Paraná). A CP02 (Fig. 5b) apresenta variações na escala de 3 a 6 meses com picos nos anos de 2005, 2007 e 2010.
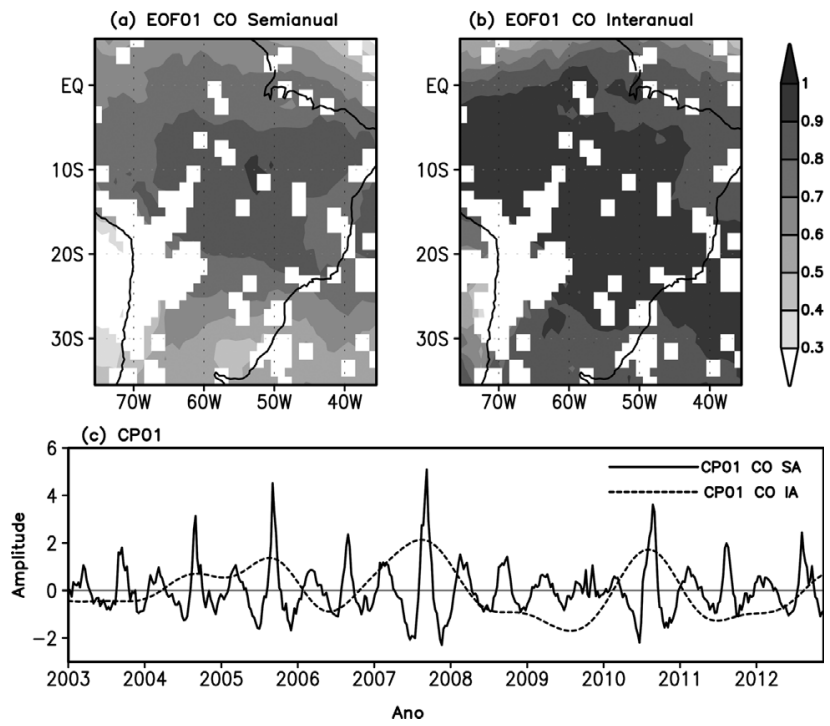

Figura 4 - Primeiro modo de EOF para séries de anomalias de CO filtradas para o período de 2003-2012: (a) padrão espacial para a escala semianual, (b) padrão espacial para a escala interanual e (c) correspondentes componentes principais.
O primeiro modo de variabilidade de $\mathrm{CO}$ na escala de tempo interanual (Fig. 4b) explica $77 \%$ da variabilidade total de $\mathrm{CO}$ contida nesta escala e é dominado por um padrão sobre toda a área de estudo, com o máximo na região central desta área e que se estende de noroeste a sudeste sobre esta área. Com correlação acima de 0,9 , este modo explica aproximadamente $81 \%$ da variância local. A CP01 deste modo exibe uma oscilação interanual com valores negativos (positivos) nos anos de 2006, 2008-2009, 2011-2012 (2005, 2007 e 2010). Similar à escala semianual, há um aumento na concentração de CO para os anos de 2005, 2007 e 2010 na escala interanual. O segundo modo interanual, explica $7,3 \%$ da variabilidade total de CO contida nesta escala e é caracterizado por máximas correlações positivas (negativas) sobre a região nordeste do Brasil (sul da área de estudo e sobre a Venezuela). A CP02 deste modo exibe uma oscilação interanual com valores positivos (negativos) nos anos de 2004 a meados de 2006, 2008, 2010 (2003, meados de 2006-07 e meados de 2009 a meados de 2010). Vale notar que os picos positivos (negativos) no início dos anos de 2004, 2006 e 2009 (2007 e 2010) coincidem com anos de La Niña (El Niño).

A fim de verificar a relação entre os modos de variabilidade interanual de $\mathrm{CO}$ e a variabilidade associada ao ENOS, foram calculadas as correlações lineares entre o índice de Oscilação Sul (IOS) e as séries de CP01 $(r=0,23)$ e CP02 ( $r=0,62)$. Estes valores de correlações indicam que o modo 1 não se relaciona simultaneamente com o ENOS. Como pode ser visto na Fig. 6, os máximos (mínimos) da CP01 antecedem picos máximos (mínimos) do IOS, ou seja, os máximos (mínimos) de CO ocorrem por volta de
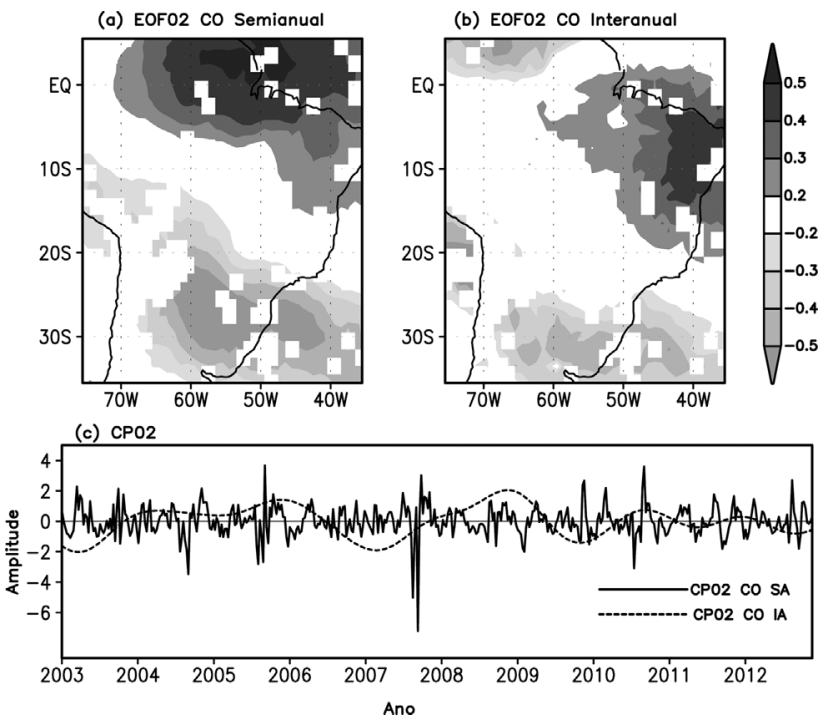

Figura 5 - Segundo modo de EOF para séries de anomalias de CO filtradas para o período de 2003-2012: (a) padrão espacial para a escala semianual, (b) padrão espacial para a escala interanual e (c) correspondentes componentes principais. 

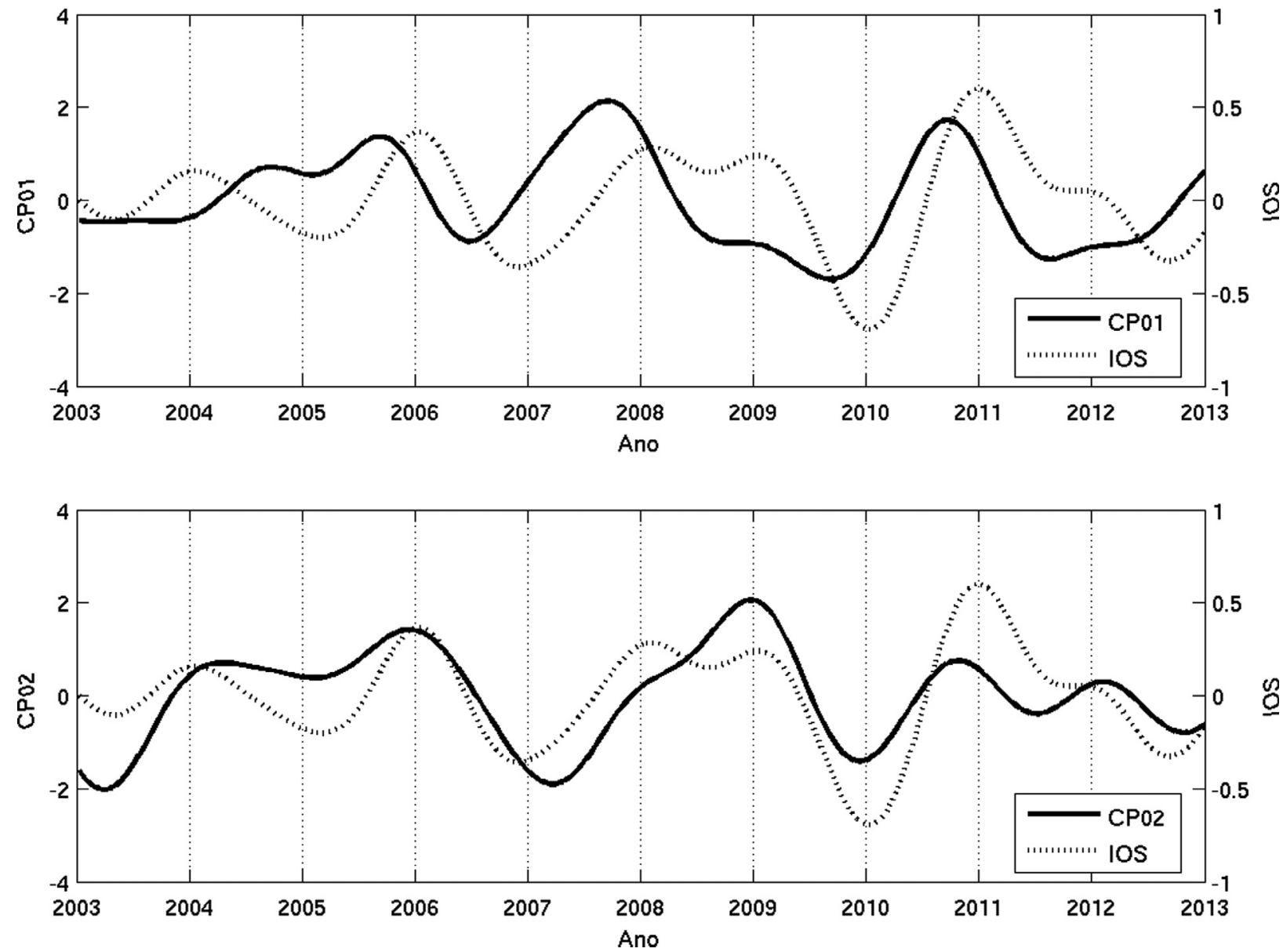

Figura 6 - Séries temporais do índice de Oscilação Sul (IOS, linha pontilhada) e das CP's correspondentes ao primeiro modo (linha contínua, painel superior) e segundo modo (linha contínua, painel inferior) das séries de $\mathrm{CO}$ filtradas para a escala interanual.

agosto a outubro durante a fase inicial de um evento La Niña (El Niño). Além disso, a Fig. 4c indica que a variabilidade interanual do $\mathrm{CO}$ pode contribuir para o aumento do $\mathrm{CO}$ na escala semianual. Por fim, a variabilidade interanual na concentração de CO na região central da América do Sul (modo 1) pode ser atribuída à variabilidade interanual da emissão de CO por queima de biomassa. Para ilustrar a relação da variabilidade interanual do $\mathrm{CO}$ (modo 1) com a queima de biomassa e precipitação construiu-se a Fig. 7. Na Fig. $7 \mathrm{a}$ a série do número de focos de queima média sobre a região entre $5^{\circ} \mathrm{S}$ e $20^{\circ} \mathrm{S}, 70^{\circ} \mathrm{W}$ e $40^{\circ} \mathrm{W}$ filtrada na escala interanual é mostrada juntamente com a CP01 interanual, e na Fig. 7b com a série de anomalias de precipitação (PRP) média para esta mesma área e também filtrada para a escala interanual. As três séries apresentam uma variabilidade interanual bem definida, com a série da CP01 oscilando quase em fase com as séries do número de focos de queima e em fase oposta com a série de precipitação. Assim, a variabilidade interanual da concentração de $\mathrm{CO}$ sobre a área de estudo notada no período de 2003 a 2012 pode ser conduzida pela emissão de CO pela queima de biomassa. Uma vez que a precipitação é o principal fator climático que controla a atividade de queima de biomassa, pode-se comprovar que a variabilidade de $\mathrm{CO}$ é influenciada por fatores climáticos, muitas vezes associados às secas como pode ser visto no grande aumento nas emissões de $\mathrm{CO}$ por queima em 2007 e 2010.

A Fig. 8 ilustra as anomalias da concentração de $\mathrm{CO}$ para setembro dos anos de 2007 e 2009, respectivamente, enquanto a Fig. 9 mostra os correspondentes mapas de anomalias de precipitação para os mesmos períodos. Assim, o aumento (diminuição) de concentração de $\mathrm{CO}$ em setembro de 2007 (2009) esteve associado ao aumento (diminuição) da emissão devido ao aumento (diminuição) do número de focos de queimada e a variabilidade climática, associada à escassez (excesso) de chuva em relação à média climatológica do período de estudo.

Por outro lado, o modo 2 descreve variações interanuais de $\mathrm{CO}$, em parte relacionadas simultaneamente ao ENOS. Assim, máximas variações interanuais de CO sobre a América do Sul equatorial nos primeiros meses do ano (Fig. 5b) ocorrem entre as latitudes de $10^{\circ} \mathrm{S}$ a $5^{\circ} \mathrm{N}$ e são resultados dos incêndios que ocorrem nas regiões norte e nordeste da América do Sul. Assim, como discutido para o modo 1 , a queima de biomassa associada à variabilidade climática pode modular a variabilidade interanual do $\mathrm{CO}$ 

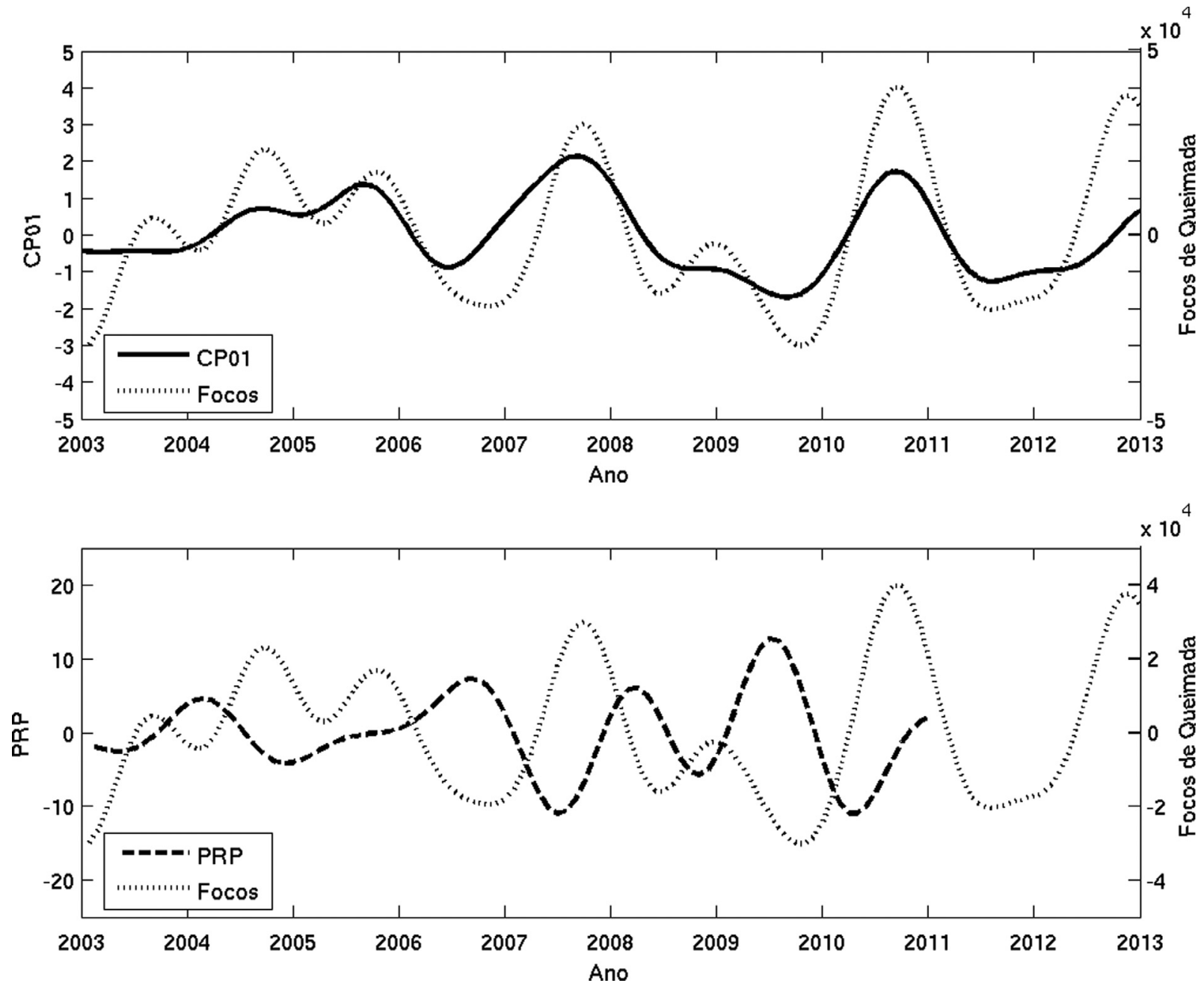

Figura 7 - (a) Séries temporais de anomalias de focos de queima (linha pontilhada) e CP01 das séries de CO filtradas para a escala interanual (linha contínua) e (b): série de anomalias de focos de queima (linha pontilhada) e anomalias de precipitação (linha tracejada) sobre a América do Sul, entre $5^{\circ} \mathrm{S}$ e $20^{\circ} \mathrm{S}$.

nessa região. A Fig. 10 mostra os mapas de anomalias de precipitação para os meses de janeiro de 2006 e fevereiro de 2007, que correspondem a dois anos contrastantes em termos de variabilidade do $\mathrm{CO}$ (Figs. 5b e c). Um aumento (Uma diminuição) de chuvas na parte norte (nordeste) da América do Sul durante o mês de janeiro de 2006 (fevereiro de 2007) associado (a) ao evento de La Niña (El Niño), está associado (a) à (ao) diminuição (aumento) do CO na região norte (nordeste) da América do Sul. Esses resultados corroboram que o ENOS em sua fase madura tem um importante efeito na variabilidade interanual de $\mathrm{CO}$ sobre a região norte e nordeste da América do Sul.

\section{Conclusões}

Neste estudo, o comportamento sazonal e os modos dominantes da variabilidade semianual e interanual da concentração de CO integrado na atmosfera sobre a América do Sul foram investigados, a partir de estimativas por satélite EOS/AQUA. Em relação ao comportamento sazonal, consistente com trabalhos anteriores, as maiores concentrações de $\mathrm{CO}$ ocorrem no período de agosto a outubro, associadas, principalmente, à queima de biomassa. Também, as concentrações mais elevadas foram notadas predominantemente na faixa latitudinal de $5^{\circ} \mathrm{S}$ a $20^{\circ} \mathrm{S}$.

Em termos de variabilidade interanual, a concentração de CO na região central da América do Sul está diretamente associada à variabilidade interanual de focos de queima na região. Maiores valores de concentração de $\mathrm{CO}$ ocorrem nas estações de grande queima com maiores números de focos nos anos de 2007 e 2010. Por outro lado, baixas concentrações ocorreram em 2008 e 2009. Uma vez que a precipitação é o principal fator climático que controla a atividade de queima de biomassa, a variabilidade de CO é influenciada por fatores climáticos, muitas vezes associados à secas como pode ser visto no grande aumento nas emissões de CO por queima em 2007 e 2010. O aumento (A diminuição) de concentração de CO no ano de 2007 (2009) esteve associado (a) ao aumento (à diminuição) da emissão, 
a) Co Setembro (2007)

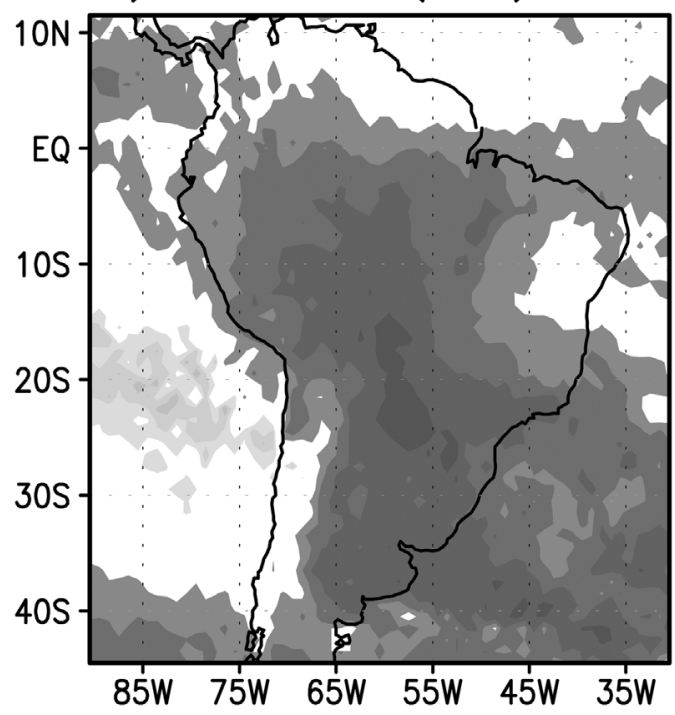

b) CO Setembro(2009)

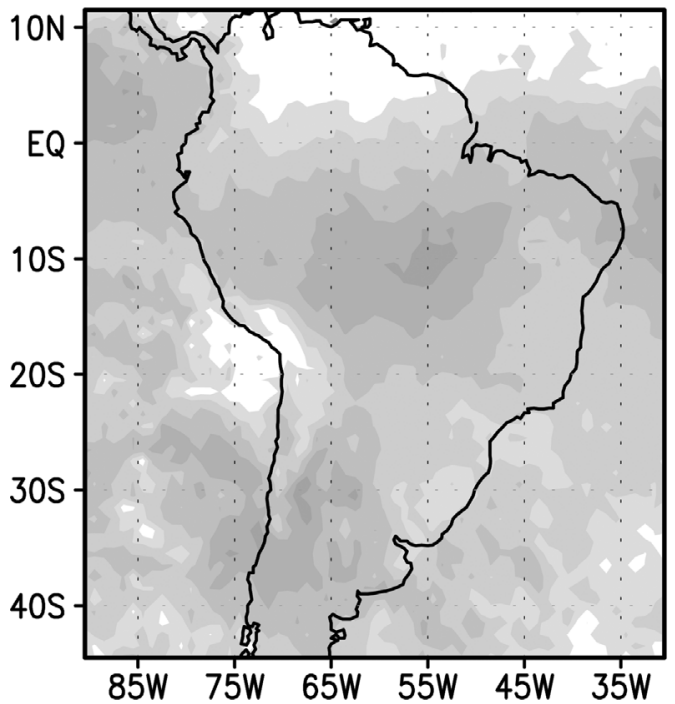

Figura 8 - Padrões espaciais de anomalias normalizadas pelo desvio padrão da concentração de CO para: (a) setembro de 2007 e (b) setembro de 2009.

devido ao aumento (à diminuição) do número de focos de queimada, associado à escassez (ao excesso) de chuva em relação à média climatológica do período de estudo. A intensa atividade de desflorestamento e consequente emissão de gases e partículas decorrentes das queimadas durante a estação seca têm implicações importantes em nível local, regional e global (Artaxo et al., 2005). Por exemplo, em anos muito secos, como os de 2007 e 2010, a queima de biomassa pode facilmente sair do controle, devido à vegetação excessivamente seca, conduzindo a elevadas emissões de CO. Além disso, a combustão continuada das queimadas libertam grandes quantidades de CO para a atmosfera (Lewis et al., 2011). Em contraste, durante anos úmidos tal como em 2009, a queima de biomassa devido ao desma- a) PRP Setembro (2007)

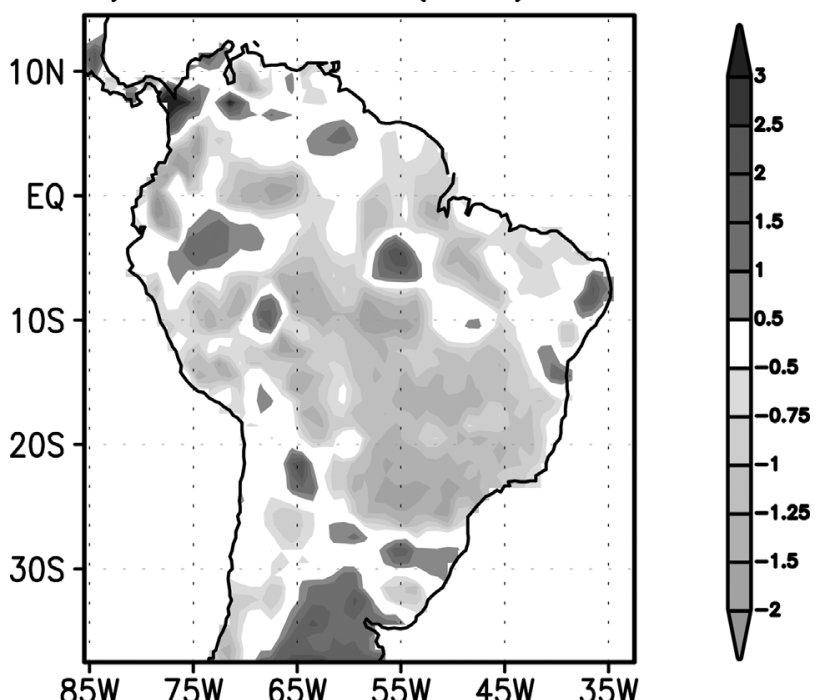

b) PRP Setembro(2009)

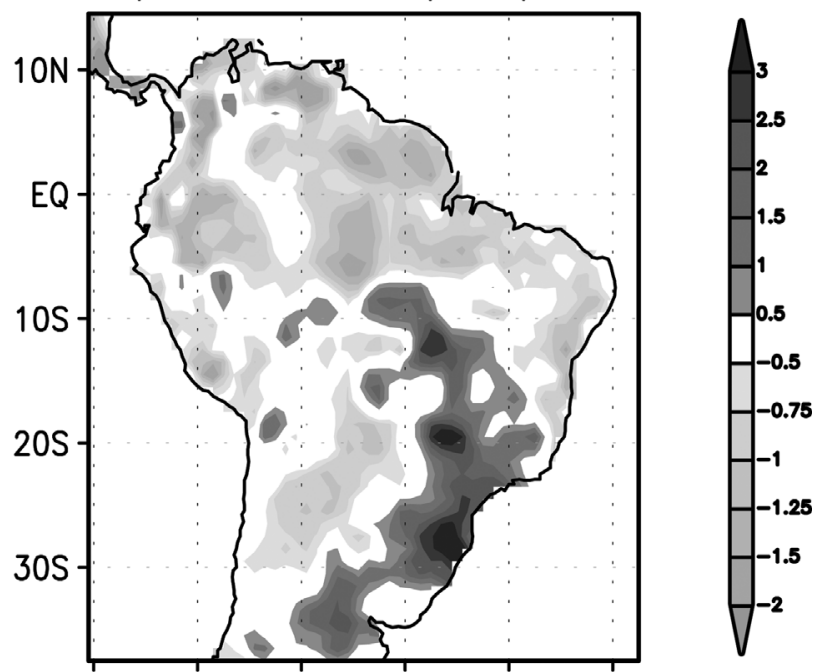

Figura 9 - Padrões espaciais de anomalias de precipitação normalizadas pelo desvio padrão para: (a) setembro de 2007 e (b) setembro de 2009.

tamento pode ser adiada devido à grande quantidade de umidade (Torres et al., 2010). Este fato pode ter levado a uma redução da emissão de $\mathrm{CO}$ neste ano. Ainda, a queima de biomassa na escala interanual está diretamente associada com a variabilidade climática. De modo que, a diminuição (aumento) de chuvas na parte central e leste da América do Sul durante a fase inicial do evento de La Niña (El Niño) contribui para aumentar (diminuir) os focos de queimada nessa região e consequentemente, as emissões de CO. Por outro lado, durante a fase madura do ENOS, a maior variabilidade acontece na região norte e nordeste da América do Sul. Esses resultados sugerem que o ENOS tem um importante efeito na variabilidade interanual de CO sobre a América do Sul. 


\section{a) PRP Janeiro (2006)}

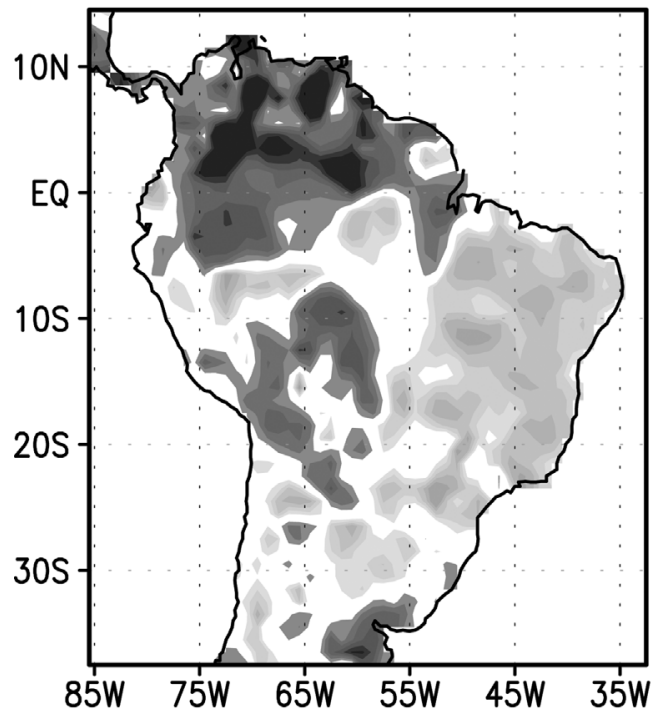

b) PRP Fevereiro(2007)

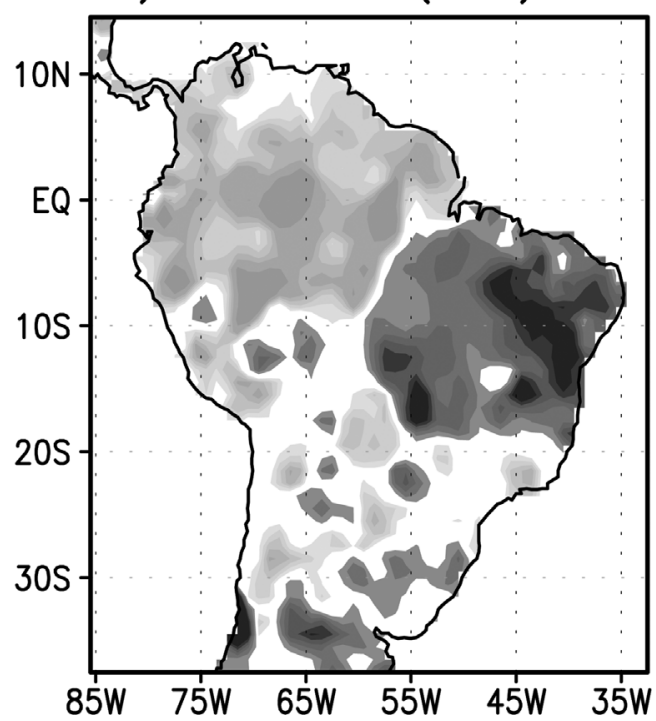

Figura 10 - Padrões espaciais de anomalias de precipitação normalizadas pelo desvio padrão para: (a) janeiro de 2006 e (b) fevereiro de 2007.

\section{Agradecimentos}

Este trabalho é parte da dissertação de Mestrado da primeira autora desenvolvida no Programa de Pós-Graduação em Clima e Ambiente do INPA/UEA, com bolsa de estudo concedida pela Coordenação de Aperfeiçoamento de Pessoal (CAPES). Os autores agradecem a FINEP e a FAPEAM (Projetos PROESTADO e GOAMAZON), pelo apoio financeiro à pesquisa. $\mathrm{O}$ quarto e quinto autores foram parcialmente financiados pelo CNPq (Processos 305942/2015-8 e 302679/2013-8, respectivamente). Os dois últimos autores agradecem ao CNPq e CAPES, respectivamente, pela concessão de bolsa de estudo. Os autores agradecem as sugestões do revisor anônimo.

\section{Referências}

ANDREAE, M.O.; ARTAXO, P.; BECK, V.; BELA, M.; FREITAS, S.; GERBIG, C.; LONGO, K.; MUNGER, J.W.; WIEDEMANN, K.T.; WOFSY, S.C. Carbon monoxide and related trace gases and aerosols over the Amazon Basin during the wet and dry seasons. Atmospheric Chemistry Physics, v. 12, p. 6041-6065, 2012.

ARTAXO, P.; GATTI, L.V.; LEAL, A.M.C.; LONGO, K.M.; FREITAS, S.R.; LARA, L.L.; PAULIQUEVIS, T.M.; PROCÓPIO, A.S.; RIZZO, L.V. Química atmosférica na Amazônia: A floresta e as emissões de queimadas controlando a composição da atmosfera amazônica. Acta Amazonica, v. 35, p. 185-196, 2005.

AUMANN, H.H.; MILLER, C. Atmospheric Infrared Sounder (AIRS) on the Earth Observing System. Proceedings SPIE, Paris, v. 2583, p. 332-343, 1995.

AUMANN, H.H.; PAGANO, R.J. Atmospheric Infrared Sounder on the Earth Observing System. Optical Engineering, v. 333, p. 776-784, 1994.

CHAHINE, M.T. (Coord.); FETZER, E. (Ed.); GUNSON, M.; AUMANN, H.; STROW, L.; HAGAN, D.; HOFSTADTER, M.; OLSEN, E.; SUSSKIND, J.; ROSENKRANZ, P.; REVERCOMB, H.; MCMILLIN, L.; GAUTIER, C.; STAELIN, D.; HUANG, A.; TOBIN, D. AIRS Algorithm Theoretical Basis Document - The AIRS team science data validation plan, Level 2. JPL D-16822. Version 2.1.1. Pasadena, Calif.: NASA. Jun, 2000. Disponível em: http:/http://eospso.gsfc.nasa.gov/sites/default/files/ atbd/AIRSValP2doc.pdf. Acesso em: 10 jan. 2014.

CONNORS, V.S.; GORMSEN, B.B.; NOLF, S.; REICHLE JUNIOR, H.G. Spaceborne observations of the global distribution of carbon monoxide in the middle troposphere during April and October 1994. Journal of Geophysical Research, v. 104, p. 21455-21470, 1999.

D'AMELIO, M.T.S. Estudo de gases de efeito estufa na Amazônia. 2006. Dissertação de Mestrado - Instituto de Pesquisas Energéticas e Nucleares - IPEN/CNEN-SP, 2006, São Paulo. Disponível em: http://http://www.teses.usp.br/teses/ disponiveis/85/85134/tde-22052007-141134/pt-br.php. Acesso em: 17 jan. 2015.

DUNCAN, B.N.; LOGAN, J.A. Model analysis of the factors regulating the trends and variability of carbon monoxide between 1988 and 1997. Atmospheric Chemistry Physics, v. 8, p. 7389-7403, 2008.

EDWARDS, D.P.; EMMONS, L.K.; GILLE, J.C.; CHU, A.; ATTIÉ, J.-L.; GIGLIO, L.; WOOD, S.W.; HAYWOOD, J.; DEETER, M.N.; MASSIE, S.T.; ZISKIN, D.C.; DRUMMOND, J.R. Satellite-observed pollution from Southern Hemisphere biomass burning. Journal of Geophysical Research, v. 111, p .1-17, 2006a.

EDWARDS, D.P.; PÉTRON, G.; NOVELLI, P.C.; EMMONS, L.K.; GILLE, J.C.; DRUMMOND, J.R. Southern Hemisphere carbon monoxide interannual variability observed by Terra/Measurement of Pollution in the Troposphere (MOPITT). Journal of Geophysical Research, v. 111, p. 1-9, 2006b.

FEARNSIDE, P.M. Deforestation in Brazilian Amazonia: History, rates and consequences, Conservation Biology, v. 19, p. 680-688, 2005.

FISHBEIN, E.; FARMER, C.B.; GRANGER, S.L.; GREGORICH, D.T.; GUNSON, M.R.; HANNON, S.E.; 
HOFSTADTER, M.D.; LEE, S.-Y.; LEROY, S.S.; STROW, L.L. Formulation and validation of simulated data for the Atmospheric Infrared Sounder (AIRS). IEEE Transactions on Geoscience Remote Sensing, v. 41, p. 314-329, 2003

HOOGHIEMSTRA, P.B.; KROL, M.C.; VAN LEEUWEN, T.T.; VAN DER WERF, G.R.; NOVELLI, P.C.; DEETER, M.N.; ABEN, I.; RÖCKMANN, T. Interannual variability of carbon monoxide emission estimates over South America from 2006 to 2010. Journal of Geophysical Research, v. 117, p. $1-15,2012$.

KUTSBACH, J.E. Empirical eigenvectors of sea-level-pressure, surface temperature and precipitation complexes over North America. Journal of Applied Meteorology, v.6 , p. 791-802, 1967

LANGENFELDS, R.L.; FRANCEY, R.J.; PAK, B.C.; STEELE, L.P.; LLOYD, J.; TRUDINGER, C.M.; ALLISON, C.E. Interannual growth rate variations of atmospheric $\mathrm{CO} 2$ and its $\delta 13 \mathrm{C}, \mathrm{H} 2, \mathrm{CH} 4$, and $\mathrm{CO}$ between 1992 and 1999 linked to biomass burning. Global Biogeochemical Cycles, v. 16, p. 1048, 2002.

LEWIS, S.L.; BRANDO, P.M.; PHILLIPS, O.L.; VAN DER HEIJDEN, G.M.F.; NEPSTAD, D. The 2010 Amazon drought. Science, v. 331, p. 554, 2011.

LIOU, K.N. An Introduction to Atmospheric Radiation. 2.ed. San Diego : Academic Press, 2002. 583p.

LIU, J.; LOGAN, J.A.; MURRAY, L.T.; PUMPHREY, H.C.; SCHWARTZ, M.J.; MEGRETSKAIA, I. A. Transport analysis and source attribution of seasonal and interannual variability of $\mathrm{CO}$ in the tropical upper troposphere and lower stratosphere. Atmospheric Chemistry Physics, v. 13, p. 129-146, 2013.

Malhi, Y., Roberts, J.T.; Betts, R.A.; Killeen, T.J.; Li, W.; Nobre, C.A. Climate change, deforestation, and the fate of the Amazon. Science, v. 319, p. 169-172, 2008.

MCMILLAN, W.W.; BARNET, C.; STROW, L.; CHAHINE, M.T.; MCCOURT, M.L.;

WARNER, J.X.; NOVELLI, P.C.; KORONTZI, S.; MADDY, E.S.; DATTA, S. Daily global maps of carbon monoxide from NASA's Atmospheric InfraRed Sounder. Geophysical Research Letters, v. 32, p. L11801, 2005.

MCMILLAN, W. W.; YURGANOV, L.; EVANS, K. E.; BARNET, C. Global Climatology of Tropospheric CO from the Atmospheric InfraRed Sounder (AIRS). 20th Conference on Climate Variability and Change 5B, p. 217-228, 2007.

MCMILLAN, W.W.; WARNER, J.X.; COMER, M.M.; MADDY, E.; CHU, A.; SPARLING, L.; ELORANTA, E.; HOFF, R.; SACHSE, G.; BARNET, C.; RAZENKOV, I.; WOLF, W. AIRS views transport from 12 to 22 July 2004 Alaskan/Canadian fires: Correlation of AIRS CO and MODIS AOD with forward trajectories and comparison of AIRS CO retrievals with DC-8 in situ measurements during INTEX-A/ICARTT. Journal of Geophysical Research, v. 113, p. D20301, 2008.

MORSE, P.; BATES, J.C.; MILLER, C.R.; CHAHINE, M.T.; O'CALLAGHAN, F.; AUMANN, H.H.; KARNIK, A.R. Development and test of the Atmospheric Infrared Sounder (AIRS) for the NASA Earth Observing System (EOS). Proceedings SPIE, v. 3759, p. 281-292, 1999.

NOVELLI, P.C.; MASARIE, K.A.; LANG, P.M.; HALL, B.D.; MYERS, R.C.; ELKINS, J.W. Reanalysis of tropospheric
CO trends: Effects of the 1997-1998 wildfires. Journal of Geophysical Research, v. 108, p. 4464, 2003.

PARKINSON, C.L. Aqua: an Earth-Observing satellite mission to examine water and other climate variables. IEEE Transactions on Geoscience Remote Sensing, v. 41, p. 173-183, 2003.

RAJAB, J.M.; TAN, K.C.; LIM, H.S.; MATJAFRI, M.Z. Investigation on the Carbon Monoxide Pollution over Peninsular Malaysia Caused by Indonesia Forest Fires from AIRS Daily Measurement. In: NEJADKOORKI, F. (Ed.) Advanced Air Pollution. Penang: InTech, 2011. Cap.8, p. $115-136$

RODGERS, C.D. Information content and optimisation of high spectral resolution remote measurements. Advances in Space Research, v. 21, p. 361-367, 1998.

SCHNEIDER, U.; FUCHS, T.; MEYER-CHRISTOFFER, A.; RUDOLF, B. Global Precipitation Analysis Products of the GPCC, Germany: Global Precipitation Climatology Centre (GPCC), 2008. 2p.

SUSSKIND, J.; BARNET, C.; BLAISDELL, J. Retrieval of atmospheric and surface parameters from AIRS/AMSU/HSB data in the presence of clouds. IEEE Transactions on Geoscience Remote Sensing, v. 41, p. 390-409, 2003.

THONAT, T.; CREVOISIER, C.; SCOTT, N. A.; CHÉDIN, A.; SCHUCK, T.; ARMANTE, R.; CRÉPEAU, L. Retrieval of tropospheric CO column from hyperspectral infrared sounders - application to four years of Aqua/AIRS and MetOp-A/IASI. Atmospheric Measurement Techniques, v.5, p. 2413-2429, 2012.

TORRENCE, C.; COMPO, G. P. A Practical Guide to Wavelet Analysis. Bulletin of the American Meteorological Society, v. 79, p. 61-78, 1998.

TORRES, O.; CHEN, Z.; JETHVA, H.; AHN, C.; FREITAS, S.R.; BHARTIA, P.K. OMI and MODIS observations of the anomalous 2008-2009 Southern Hemisphere biomass burning seasons. Atmospheric Chemistry Physics, v. 10, p. 3505-3513, 2010.

WALLACE, J.M.; HOBBS, P.V. Atmospheric Science: An Introductory Survey. 2 ed. San Diego : Academic Press, 2006. $504 \mathrm{p}$.

WOTAWA, G.; NOVELLI, P.C.; TRAINER, M.; GRANIER, C. Inter-annual variability of summertime $\mathrm{CO}$ concentrations in the Northern Hemisphere explained by boreal forest fires in North America and Russia. Geophysical Research Letters, v. 28, p. 4575-4578, 2001.

YURGANOV, L.N.; MCMILLAN, W.W.; DZHOLA，A.V.; GRECHKO, E.I.;

JONES, N.B.; VAN DER WERF, G.R. Global AIRS and MOPITT CO measurements: Validation, comparison, and links to biomass burning variations and carbon cycle. Journal of Geophysical Research, v. 113, p. D09301, 2008.

\section{Recursos de Internet}

Portal da NASA, http://airs.jpl.nasa.gov/.

Índice de Oscilação Sul. Disponível em: https://www.longpaddock.qld.gov.au/seasonalclimateoutlo ok/southernoscillationindex/soidatafiles/DailySOI1933-19 92Base.txt.

This is an Open Access article distributed under the terms of the Creative Commons Attribution Non-Commercial License which permits unrestricted non-commercial use, distribution, and reproduction in any medium provided the original work is properly cited. 